\title{
Impact of third-line treatment with irinotecan plus cetuximab on non-tumor standardized uptake values in patients with metastatic colorectal cancer
}

\author{
KIM FRANCIS ANDERSEN ${ }^{1,2}$, KRISTIN SKOUGAARD ${ }^{1,3}$, ANNE LERBERG NIELSEN $^{4}$ \\ and HELLE WESTERGREN HENDEL ${ }^{1}$ \\ ${ }^{1}$ Department of Clinical Physiology and Nuclear Medicine, Herlev Hospital, DK-2730 Herlev; \\ ${ }^{2}$ Department of Clinical Physiology and Nuclear Medicine, Bispebjerg Hospital, DK-2400 Copenhagen NW; \\ ${ }^{3}$ Department of Oncology, Herlev Hospital, University Hospital of Copenhagen, DK-2730 Herlev; \\ ${ }^{4}$ Department of Nuclear Medicine, Odense University Hospital, DK-5000 Odense C, Denmark
}

Received January 31, 2012; Accepted April 2, 2012

DOI: $10.3892 / \mathrm{ol} .2012 .683$

\begin{abstract}
The correct interpretation of metabolic response in cancer cells to therapy requires knowledge of how tumor-free tissue responds to the same treatment. The aim of this study was to evaluate standardized uptake values (SUVs) in tumor-free regions of patients with metastatic colorectal cancer prior to and following therapy, via the use of 18-fluoride fluoro-2-deoxy-D-glucose (18F-FDG) positron emission tomography/computed tomography (PET/CT). On baseline 18F-FDG PET/CT scans $(n=51)$, volumes of interest (VOI) were obtained from tumor-free tissue (aortic arch, liver and spleen) and SUVs normalized to total body mass were registered. The procedure was repeated for a follow-up scan two weeks following a single administration of the thirdline treatment with irinotecan plus cetuximab. The mean differences in SUV prior to and following therapy were nonsignificant $(\mathrm{P}>0.05)$ in all the registered tumor-free regions. Correlation coefficients indicated a significant result between the variables $(0.74-0.84 ; \mathrm{P}<0.001)$. This study suggests that the early assessment of metabolic response may be made following the administration of third-line therapy with irinotecan plus cetuximab in patients with metastatic colorectal cancer refractory to second-line treatment with irinotecan.
\end{abstract}

Correspondence to: Dr Kim Francis Andersen, Department of Clinical Physiology and Nuclear Medicine, Bispebjerg Hospital, University Hospital of Copenhagen, Bispebjerg Bakke 23, Entrance 60, Floor A, DK-2400 Copenhagen NW, Denmark E-mail: kand0236@bbh.regionh.dk

Key words: early assessment, metabolic response, metastatic colorectal cancer, non-tumor tissue, standardized uptake values

\section{Introduction}

With an estimated incidence of 333,330 cases in the European Union (2008) and reports of high mortality rates, colorectal cancer (CRC) is one of the most common causes of cancer-related mortality in the US and Europe (1). Metastatic disease is present in approximately $25 \%$ of patients at the time of diagnosis, with $50 \%$ of patients likely to develop metastases. The majority of these patients are unlikely to be available for surgical resection with a primary curative intention.

Therapy for advanced cancer, either on an individual basis or via clinical trials, may often be toxic to the patient and also costly and response rates are considered to be relatively low. The timely discontinuation of treatment is therefore crucial. The decision to continue, alter or terminate a specific treatment regimen is often based upon morphological imaging. The RECIST criteria $(2,3)$ have, until the introduction of positron emission tomography (PET), been used as a 'gold standard' for response evaluation. Imaging of glucose metabolism in cancer cells with quantitative PET applying the glucose analog 18-fluoride fluoro-2-deoxy-D-glucose (18F-FDG), has emerged as a powerful tool (4-6), with numerous studies reporting a positive correlation between the tumor $18 \mathrm{~F}-\mathrm{FDG}$ uptake immediately following or during treatment and the clinical outcome. Changes in tumor metabolism may be observed prior to changes in tumor size, providing information that may be used for early individual risk assessment or as an early surrogate endpoint in a clinical trial.

The metabolic response on an 18F-FDG PET scan may be determined using qualitative and quantitative approaches. The most common method for quantifying FDG uptake is via the application of standardized uptake values (SUVs), which may be normalized to body mass, lean body mass or body surface area. Being relatively easy to access, SUVs have gained popularity in the clinic. Standardized protocols, including patient preparation, scanning procedure, image reconstruction and image analysis, are essential when patients are studied over a period of time or are participating in multicenter studies (7-10). 
As changes in plasma glucose levels and/or differences in FDG plasma clearance among scans may interfere with the interpretation of SUV results $(11,12)$, factors affecting these parameters should also be standardized. The European Organisation for Research and Treatment of Cancer (EORTC), the National Cancer Institute (NCI) and the European Association of Nuclear Medicine have all made consensus recommendations with regard to SUV and the issues mentioned above $(9,13,14)$. Although these recommendations are followed, studies assessing metabolic response to therapy also have to address the following consideration: SUVs in reproducible, tumor-free regions of interest (ROI) should be significantly consistent throughout therapy, therefore a significant change in tumor SUV would be indicative of a therapeutic metabolic response.

By using 18F-FDG PET/CT, the aim of this study was to evaluate SUVs in non-tumor volumes of interest (VOI) in patients with metastatic CRC refractory to second-line treatment with irinotecan just prior to and 2 weeks following a single administration of third-line therapy with irinotecan plus cetuximab.

\section{Materials and methods}

Selection and description of participants. The study occurred at a specialist cancer treatment center. Only patients with metastatic CRC participated in the study. The inclusion and exclusion criteria are listed in Fig. 1. A total of 51 patients (mean body weight, $74 \pm 18 \mathrm{~kg}$; range, $47-132 \mathrm{~kg}$; follow-up, $74 \pm 17 \mathrm{~kg}$, range, $47-130 \mathrm{~kg}$ ) underwent a baseline 18F-FDG $\mathrm{PET} / \mathrm{CT}$ scan prior to a single administration of irinotecan $\left(180 \mathrm{mg} / \mathrm{m}^{2}\right)$ plus cetuximab $\left(500 \mathrm{mg} / \mathrm{m}^{2}\right)$. A follow-up scan was performed at two weeks following treatment. The procedure followed was according to a protocol approved by the Regional Ethics Committee of Copenhagen County and with the Helsinki declaration (2008). Oral and written informed consent from the patients were obtained prior to any patient participating in the study.

Methods.Adose of $4 \mathrm{MBq} / \mathrm{kg}$ (maximum400MBq)of 18F-FDG was injected intravenously (i.v.) following a minimum 6-h fast in patients with blood glucose levels $<120 \mathrm{mg} / \mathrm{dl}$. $\mathrm{PET} / \mathrm{CT}$ data were acquired at $60 \mathrm{~min}$ post-injection (p.i.) on a GE Healthcare Discovery ${ }^{\mathrm{TM}}$ (Buckinghamshire, UK) VCT PET/CT scanner (15). A helical diagnostic CT scan was acquired with oral (E-Z-Cat ${ }^{\circledR} 0.91$ solution) and i.v. contrast (Ultravist ${ }^{\circledR} 370 \mathrm{mg} \mathrm{I} / \mathrm{ml}$ ) using a standard CT protocol with a scan field of view of $70 \mathrm{~cm}$. Data were reconstructed with a standard filter into transaxial slices with a field of view of $50 \mathrm{~cm}$, matrix size of $512 \times 512$ (pixel size $0.98 \mathrm{~mm}$ ) and a slice thickness of $3.75 \mathrm{~mm}$. The CT scan was followed immediately by a PET scan performed using a standard whole-body acquisition protocol with 6-7 bed positions, a slice overlap of 7 and an acquisition time of 2.5 min per bed position. The scan field of view was $70 \mathrm{~cm}$. The attenuation correction was based on the CT scan. The PET data were reconstructed into transaxial slices with a matrix size of $128 \times 128$ (pixel size $5.47 \mathrm{~mm}$ ) and a slice thickness of $3.75 \mathrm{~mm}$ using iterative 3D-OSEM (2 iterations, 28 subsets). Corrections for attenuation, randoms, dead time and normalization were carried out inside the iterative loop. Analyses of

\section{Inclusion criteria: \\ - $\quad$ Age $\geq 18$ years \\ - Histologically proven, irinotecan-resistant, metastatic colorectal cancer \\ - Former treatment with oxaliplatin \\ - Performance status (WHO) $<3$ \\ - Life expectancy $>3$ months \\ - Normal hematological values (neutrophil granulocytes, thrombocytes, bilirubin and ASAT/ALAT) \\ - Oral/written informed consent}

\section{Exclusion criteria:}

- Age $<18$ years

- Former or concurrent malignancy

- Pregnant and/or lactating women

- Patients not able to follow treatment and evaluation schemes

- Ongoing infection and/or concurrent serious medical illness

- Known hypersensitivity to any of the components in the treatment scheme

- Abnormal hematological values

- Lack of oral/written informed consent

Figure 1. Criteria for inclusion and exclusion.

CT, PET and fused PET/CT data were performed using a GE Healthcare Volume Viewer® on a GE Healthcare Advantage Workstation ${ }^{\circledR}$ version 4.4. Approximately $10 \mathrm{~cm}^{3}$ VOI was drawn in the aortic arch, in tumor-free liver and in the spleen and SUVs (maximum and mean) normalized to total body mass were registered for all regions. Baseline and follow-up data were obtained using the same PET/CT scanner. To avoid possible inter-observer bias, the same physician analyzed all scans $(16,17)$.

Statistical analysis. Calculations of sample size and power were performed using the Altman nomogram (18). The minimal relevant difference (MIREDIF) in SUV was set to be equal to the standard deviation, yielding a standardized difference of 1.0. The power of the study was determined to be 0.94 . Any other statistical analyses were performed using MedCalc 11.1.1 ${ }^{\circledR}$ (Mariakerke, Belgium) and SPSS Statistics $17.0^{\circledR}$ (Chicago, IL, USA). The SUV results were compared with Gaussian distributions by applying the D'AgostinoPearson omnibus test $(19,20)$. The SUV measurements passed the test for normality $(\mathrm{P}>0.05)$. Paired samples t-test was used to compare two sets of results to assess whether there was any difference between the means. Correlation coefficients were calculated to measure the strength of correlation between variables. $\mathrm{P}<0.05$ was considered to indicate a statistically significant result.

\section{Results}

The results of this study showed significantly consistent SUVs $\left(\mathrm{SUV}_{\text {max }}\right.$ and $\left.\mathrm{SUV}_{\text {mean }}\right)$ in the aortic arch, liver and spleen 
Table I. Baseline SUVs prior to treatment.

\begin{tabular}{|c|c|c|c|}
\hline Region (10 $\mathrm{cm}^{3}$ VOI) & Mean $\pm \mathrm{SD}$ & CI $(95 \%)$ & Normal distribution (P-value) \\
\hline Aortic arch $\mathrm{SUV}_{\max }$ & $1.66 \pm 0.37$ & $1.55-1.76$ & 0.66 \\
\hline Aortic arch $\mathrm{SUV}_{\text {mean }}$ & $1.18 \pm 0.26$ & $1.10-1.25$ & 0.66 \\
\hline Liver $\mathrm{SUV}_{\max }$ & $2.13 \pm 0.50$ & $1.99-2.27$ & 0.16 \\
\hline Liver $\mathrm{SUV}_{\text {mean }}$ & $1.58 \pm 0.34$ & $1.49-1.68$ & 0.62 \\
\hline Spleen $\mathrm{SUV}_{\max }$ & $1.83 \pm 0.48$ & $1.70-1.97$ & 0.07 \\
\hline Spleen $\mathrm{SUV}_{\text {mean }}$ & $1.35 \pm 0.36$ & $1.25-1.45$ & 0.33 \\
\hline
\end{tabular}

$\mathrm{N}=51 . \mathrm{P}<0.05$ was considered to indicate a statistically significant result. Data were tested for normal distribution using the D'Agostino-Pearson omnibus test. SUV, standardized uptake value; SD, standard deviation; CI, confidence interval; VOI, volume of interest.

Table II. SUVs following treatment.

\begin{tabular}{lll}
\hline Region $\left(10 \mathrm{~cm}^{3} \mathrm{VOI}\right)$ & Mean $\pm \mathrm{SD}$ & CI $(95 \%)$ \\
\hline Aortic arch $\mathrm{SUV}_{\text {max }}$ & $1.70 \pm 0.48$ & $1.57-1.83$ \\
${\text { Aortic } \operatorname{arch~} \mathrm{SUV}_{\text {mean }}}$ & $1.20 \pm 0.34$ & $1.10-1.29$ \\
Liver SUV $_{\text {max }}$ & $2.12 \pm 0.55$ & $1.96-2.27$ \\
Liver SUV $_{\text {mean }}$ & $1.59 \pm 0.41$ & $1.47-1.71$ \\
Spleen $_{\text {SUV }}$ & $1.89 \pm 0.54$ & $1.74-2.04$ \\
Spleen $_{\text {max }}$ & $1.35 \pm 0.39$ & $1.24-1.46$ \\
\hline
\end{tabular}

$\mathrm{N}=51$. SUV, standardized uptake value; SD, standard deviation; CI, confidence interval; VOI, volume of interest.

tumor-free regions prior to and 2 weeks following a single administration of third-line treatment with irinotecan plus cetuximab in patients with irinotecan refractory metastatic CRC (Tables I and II).

The mean differences were non-significant $(\mathrm{P}>0.05$; Table III) in the aortic arch, liver and spleen regions of interest and $\mathrm{SUV}_{\max }$ and $\mathrm{SUV}_{\text {mean }}$. The correlation coefficients were significant $(\mathrm{P}<0.001$; Table III), ranging from 0.74 to 0.84 .

\section{Discussion}

Semi-quantitative analysis of glucose metabolism in tumors with 18 F-FDG PET in the prediction of clinical outcome is gaining popularity (4-6), due to the evidence that changes in tumor metabolism may be observed prior to changes in tumor size. Knowledge of how tumor-free tissue responds to the same treatment regimen is required for the correct interpretation of metabolic response in cancer cells to therapy. Thus, a significant change in tumor SUV would be indicative of a therapeutic metabolic response if SUVs in reproducible, non-tumor ROI are consistent throughout therapy. Response rates to therapy for metastatic colorectal cancer are markedly low and a swift individual assessment for different treatment regimens is crucial.

Assuming all consensus recommendations with regard to data acquisition and patient preparation are fulfilled $(9,13,14)$, this study demonstrates significantly consistent SUVs (both $\mathrm{SUV}_{\max }$ and $\mathrm{SUV}_{\text {mean }}$ ) in three different tumor-free regions (aortic arch, liver and spleen) prior to and 2 weeks following a single administration of third-line treatment with irinotecan plus cetuximab in patients with irinotecan refractory metastatic CRC. This study provides the fundamental data needed for studies focusing on the early assessment of therapeutic response in these patients with this specific treatment regimen.

Table III. Comparison of SUV prior to and following treatment (paired samples t-test and test of correlation).

\begin{tabular}{|c|c|c|c|c|c|}
\hline Region $\left(10 \mathrm{~cm}^{3} \mathrm{VOI}\right)$ & Mean difference \pm SD & CI $(95 \%)$ & P-value & Correlation & P-value \\
\hline Aortic arch $\mathrm{SUV}_{\max }$ & $-0.045 \pm 0.32$ & $-0.135-0.045$ & 0.32 & 0.74 & $<0.001^{\mathrm{a}}$ \\
\hline Aortic arch $\mathrm{SUV}_{\text {mean }}$ & $-0.022 \pm 0.23$ & $-0.086-0.042$ & 0.50 & 0.74 & $<0.001^{\mathrm{a}}$ \\
\hline Liver $\mathrm{SUV}_{\max }$ & $0.010 \pm 0.30$ & $-0.074-0.094$ & 0.82 & 0.84 & $<0.001^{\mathrm{a}}$ \\
\hline Liver $\mathrm{SUV}_{\text {mean }}$ & $-0.006 \pm 0.26$ & $-0.080-0.068$ & 0.87 & 0.77 & $<0.001^{\mathrm{a}}$ \\
\hline Spleen $\mathrm{SUV}_{\max }$ & $0.053 \pm 0.33$ & $-0.145-0.039$ & 0.25 & 0.80 & $<0.001^{\mathrm{a}}$ \\
\hline Spleen $\mathrm{SUV}_{\text {mean }}$ & $-0.004 \pm 0.24$ & $-0.072-0.064$ & 0.91 & 0.80 & $<0.001^{\mathrm{a}}$ \\
\hline
\end{tabular}

$\mathrm{N}=51 .{ }^{\mathrm{a}} \mathrm{P}<0.05$ was considered to indicate a statistically significant result. SUV, standardized uptake value; SD, standard deviation; CI, confidence interval; VOI, volume of interest. 


\section{References}

1. Ferlay J, Parkin DM and Steliarova-Foucher E: Estimates of cancer incidence and mortality in Europe in 2008. Eur J Cancer 46: 765-81, 2010.

2. Eisenhauer EA, Therasse P, Bogaerts J, Schwartz LH, Sargent D, Ford R, Dancey J, Arbuck S, Gwyther S, Mooney M, et al: New response evaluation criteria in solid tumours: revised RECIST guideline (version 1.1). Eur J Cancer 45: 228-247, 2009.

3. Therasse P, Arbuck SG, Eisenhauer EA, Wanders J, Kaplan RS, Rubinstein L, Verweij J, Van GM, van Oosterom AT, Christian MC and Gwyther SG: New guidelines to evaluate the response to treatment in solid tumors. European Organization for Research and Treatment of Cancer, National Cancer Institute of the United States, National Cancer Institute of Canada. J Natl Cancer Inst 92: 205-216, 2000.

4. Kidd EA, Siegel BA, Dehdashti F and Grigsby PW: The standardized uptake value for F-18 fluorodeoxyglucose is a sensitive predictive biomarker for cervical cancer treatment response and survival. Cancer 110: 1738-1744, 2007.

5. Larson SM and Schwartz LH: 18 F-FDG PET as a candidate for 'qualified biomarker': functional assessment of treatment response in oncology. J Nucl Med 47: 901-903, 2006.

6. Weber WA: Positron emission tomography as an imaging biomarker. J Clin Oncol 24: 3282-3292, 2006.

7. Boellaard R: Standards for PET image acquisition and quantitative data analysis. J Nucl Med 50 (Suppl 1): S11-S20, 2009.

8. Cheebsumon P, Velasquez LM, Hoekstra CJ, Hayes W, Kloet RW, Hoetjes NJ, Smit EF, Hoekstra OS, Lammertsma AA and Boellaard R: Measuring response to therapy using FDG PET: semi-quantitative and full kinetic analysis. Eur J Nucl Med Mol Imaging, January 6, 2011 (Epub ahead of print).

9. Shankar LK, Hoffman JM, Bacharach S, Graham MM, Karp J, Lammertsma AA, Larson S, Mankoff DA, Siegel BA, Van den Abbeele A, Yap J and Sullivan D: Consensus recommendations for the use of 18F-FDG PET as an indicator of therapeutic response in patients in National Cancer Institute Trials. J Nucl Med 47: 1059-1066, 2006.

10. Weber WA: Use of PET for monitoring cancer therapy and for predicting outcome. J Nucl Med 46: 983-995, 2005.
11. Freedman NM, Sundaram SK, Kurdziel K, Carrasquillo JA, Whatley M, Carson JM, Sellers D, Libutti SK, Yang JC and Bacharach SL: Comparison of SUV and Patlak slope for monitoring of cancer therapy using serial PET scans. Eur J Nucl Med Mol Imaging 30: 46-53, 2003.

12. Huang SC: Anatomy of SUV. Standardized uptake value. Nucl Med Biol 27: 643-646, 2000.

13. Boellaard R, O'Doherty MJ, Weber WA, Mottaghy FM, Lonsdale MN, Stroobants SG, Oyen WJ, Kotzerke J, Hoekstra OS, Pruim J, et al: FDG PET and PET/CT: EANM procedure guidelines for tumour PET imaging: version 1.0. Eur J Nucl Med Mol Imaging 37: 181-200, 2010.

14. Young H, Baum R, Cremerius U, Herholz K, Hoekstra O, Lammertsma AA, Pruim J and Price P: Measurement of clinical and subclinical tumour response using [18F]-fluorodeoxyglucose and positron emission tomography: review and 1999 EORTC recommendations. European Organization for Research and Treatment of Cancer (EORTC) PET Study Group. Eur J Cancer 35: 1773-1782, 1999

15. Teräs M, Tolvanen T, Johansson JJ, Williams JJ and Knuuti J: Performance of the new generation of whole-body PET/CT scanners: Discovery STE and Discovery VCT. Eur J Nucl Med Mol Imaging 34: 1683-1692, 2007.

16. Benz MR, Evilevitch V, Allen-Auerbach MS, Eilber FC, Phelps ME, Czernin J and Weber WA: Treatment monitoring by 18F-FDG PET/CT in patients with sarcomas: interobserver variability of quantitative parameters in treatment-induced changes in histopathologically responding and nonresponding tumors. J Nucl Med 49: 1038-1046, 2008

17. Marom EM, Munden RF, Truong MT, Gladish GW, Podoloff DA, Mawlawi O, Broemeling LD, Bruzzi JF and Macapinlac HA: Interobserver and intraobserver variability of standardized uptake value measurements in non-small-cell lung cancer. J Thorac Imaging 21: 205-212, 2006.

18. Altman DG: Statistics and ethics in medical research: III How large a sample? Br Med J 281: 1336-1338, 1980.

19. D'Agostino RB and Pearson ES: Tests for departure from normality. Empirical results for the distributions of $b^{2}$ and $\sqrt{ } b^{1}$. Biometrika 60: 613-622, 1973.

20. D'Agostino RB and Stephens MA (eds): Goodness-of-Fit Techniques. Marcel Dekker, New York, 1986. 